\title{
A Fast Approach for Determining of Visibility of 3D Object's Surfaces
}

\author{
Nikolai Metodiev Sirakov \\ Institute of Mechanics Bulgarian Academy of Sciences \\ Acad.G.Bonchev str. bl.4 \\ Bl.4, 1113 Sofia, Bulgaria \\ E-mail: nmsir@bgcict.acad.bg \\ Fax: + 359-2-70-20-56
}

\begin{abstract}
In the article we present an effective approach for determining of visibility of $3 \mathrm{D}$ object surfaces. This approach is suitable in the case of visualisation of complicated objects with many branches and the objects surfaces are approximated by number of triangles. Therefore to determine the visibility of 3D object surfaces, we investigate the visibility of a triangle in the 3D space. For finding of visibility of the triangles we develop an approach and algorithm based on the notions regularity and finite numerical sequences. We solve also the problem of finding the image of a closed polygon and the visibility of his edges after applying rotation. The calculation complexity of the algorithm is given. The advantages of the algorithm with respect to the others in the same field are presented in the article. We give also results of an experiment performed using this algorithm.
\end{abstract}

Key Words: Regularity, Visibility of Edges and Surfaces, 3D Objects Reconstruction, Representation, Recognition.

\section{Introduction}

The 3D reconstruction and visualisation have many practical and scientific applications in the field of Mining, Medicine Computer Vision, Robotics and Virtual Reality [16], [17 ]. The realistic visualisation of 3D objects is one important part of the problem of the 3D objects representation. A large number of methods, solving this problem, may be found in the references. An approach based on the tetra three is presented in article [13 ]. In article [1] the models of the objects are created by help of pieces from surface. In this way visible and invisible elements may be found. An algorithm working in a space of spheres and cylinders is presented in [7]. An interesting idea for searching of visible elements of $3 \mathrm{D}$ objects is proposed in [2]. There the $3 \mathrm{D}$ objects models are represented as convex shell of intersecting spheres. This approach by Sutherland [12] was improved. An method for 3D objects reconstruction and representation based on Delaenay triangulation and Voronoi diagrams is proposed in [15].

When we have to reconstruct $3 \mathrm{D}$ complex scenes other problem may also appear. Such as the problem for finding the overlapped objects and reconstruction of their hidden surfaces. The problems of observation of several 3D objects is discussed in [3]. This approach is based on a space of objects. A method for finding the intersection between 3D objects is presented in the article [5]. The problem for visibility of $3 \mathrm{D}$ object surfaces is discussed in [14]. The perception problems are solved in [6]. Most of the upper methods make a lot of calculations when solving the 
visibility problem and take a lot of computer power when the object's shape is complicated and has many branches. Something more this methods repeat the calculations each time when a rotation of the objects is made.

For solving the visibility problem of 3D objects surfaces we propose in the present article an approach based on regularities, consistencies and essential points of a closed polygon [8], [10]. By using this technique we restrict the problem of visibility to the problem of processing and compering the elements of finite numerical sequences. So we obtain the following advantages:

1. The problem for finding the visibility and the image of 3D objects surfaces after applying of rotation is reduced to the problem of processing finite numerical sequences and comparing of Integer numbers;

2. High speed of performance is obtained.

Because of these advantages our approach is suitable for application in Medical Computer Vision, Virtual Reality, Quality Control.

In the article we assume that the input data is obtained by Tomograf Computer Imagery and contain 3D co-ordinates of points from the object's surfaces [16]. With the help of these points we construct planes which contain 2D sections from the 3D objects. We assume also that all 2D objects, in each plane, are found and their borders are approximated by closed polygons. The set of all $2 \mathrm{D}$ objects is separated to subsets of "similar" 2D objects. Each subset present one 3D object.

\section{Visibility of 2D Border Points and Edges}

In the present paragraph we discuss the problem for finding the visibility of plane points in respect to some observer. The direction of the observation is parallel to the positive direction of the axis 0y (see Fig. I).

Let us assume a point $\mathrm{A}$ on the plane. Just one straight line "a" pass through point A so that this line is parallel to the axis $0 \mathrm{y}$. If point $\mathrm{B}$ lying on the line "a" exist in a way that $y_{A}>y_{B}$ we call the point $A$ invisible along the positive direction of the axis $0 y$. On the contrary, if $y_{A}<y_{B}$ the point $A$ is visible.

We base our approach on the notions regularity and consistency introduced in [8]. We approximate there the boundary of $2 \mathrm{D}$ objects by closed polygons with defined accuracy. After that we describe each polygon using consistency. As first point of this description we use the point $\left(\mathrm{x}_{\min }, \mathrm{y}_{\min }\right)$. The direction of the description is clockwise. The consistency is constructed from regularities. The notion regularity is introduced as the direction of movement of a point over a closed polygon. The plane points satisfy eight different regularities shown on Figure 1. Each regularity represents just one edge of a closed polygon.

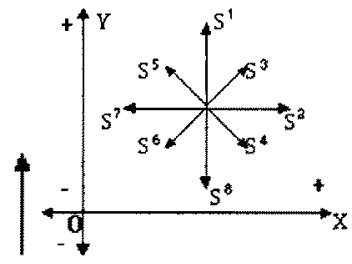

Figure 1. The regularities which plane points satisfy. 
As example, the closed polygon shown on Figure 2. is described by the consistency presented in (1). $\mathrm{By} \mathrm{Cl}$ we denote that the consistency is closed.

$$
\mathrm{S}_{\mathrm{i}}{ }^{2} \underset{i=1}{2} \mathrm{~S}_{\mathrm{i}}{ }^{3} \underset{i=3}{\stackrel{5}{\rightarrow}} \mathrm{S}_{6}{ }^{2} \rightarrow \mathrm{S}_{7}{ }^{1} \rightarrow \mathrm{S}_{8}{ }^{2} \rightarrow \mathrm{S}_{\mathrm{i}}{ }^{4} \underset{i=9}{\stackrel{10}{\rightarrow}} \mathrm{S}_{\mathrm{i}}{ }^{6} \underset{i=11}{\rightarrow 12} \mathrm{~S}_{13}{ }^{7} \rightarrow \mathrm{S}_{14}{ }^{6} \rightarrow \mathrm{Cl}
$$

Further each consistency falls apart to four finite numerical sequences:

$\mathrm{Rg}$ - the sequence of regularities constructing the consistency; $\mathrm{Rp}$ - the sequence containing repetition indicators, showing how many times each regularity from $\mathrm{Rg}$ consists of consecutively in the consistency; An -the sequence of the angles of the regularities from $\mathrm{Rg}$. Each regularity have an angle with the positive direction of the axis Ox; Le -the sequence of the lengths of the regularities from $\mathrm{Rg}$ (or sequence containing first point of each regularity).

As a result the consistency shown in (1) falls apart to four numerical sequences:

$\mathrm{Rg}=5,3,2,1,2,4,6,7,6 ; \quad \mathrm{Rp}=2,3,1,1,1,2,2,1,1 ; \quad \mathrm{An}=\varphi_{1}, \ldots, \varphi_{14} ; \quad \mathrm{Le}=\mathrm{L}_{1}, \ldots ., \mathrm{L}_{14}$.

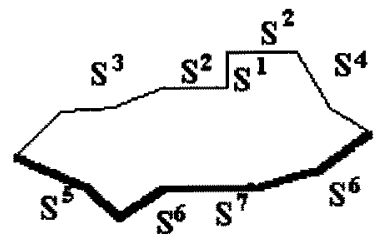

Figure 2. Closed polygon. The visible regularities are denoted by the dark line.

We consider that one regularity describes an invisible or partially covered edge of a closed polygon if at least one end of this regularity is invisible. When the pair ends are visible the regularity describes a visible edge.

Statement 1 . The regularities $S^{1}, S^{2}, S^{3}, S^{4}, S^{8}$ describe only invisible edges of closed polygon along the positive direction of the axis $0 \mathrm{y}$.

Proof: From the definition of regularity, first point and direction of polygon description follows that in each consistency for each point $A$ of the regularities $\mathrm{S}^{2}, \mathrm{~S}^{3}$ ,$S^{4}$ exists at least one point $B$ from some of the regularities $S^{5}, S^{6}, S^{7}$ so that $Y_{A}>$ $Y_{B}$. Therefore the regularities $S^{2}, S^{3}, S^{4}$ describe invisible edges of closed polygon. From the definitions of the regularities $S^{1}$ and $S^{8}$ follows that they describe invisible edges.

From Statement 1. follows that invisible edges of some closed polygon may be find by using the sequences $\mathrm{Rg}$ and $\mathrm{Rp}$ of the corresponding consistency.

Statement 2. The regularities $S^{5}, S^{6}, S^{7}$ describe visible edges if, in consistency, before or behind them the regularities $S^{1}, S^{2}, S^{3}, S^{4}, S^{8}$ do not appear.

Proof: It follows from the definition of the notion regularity, first point and direction of polygon description that in each consistency as first regularity may be only one from the regularities $S^{1}, S^{3}, S^{5}$. Also as last regularity may be only one from the regularities $S^{4}, S^{6}, S^{7}, S^{8}$. Therefore from the definitions of $S^{5}, S^{6}, S^{7}$ follows that $S^{5}$ is visible when it is in beginning and $S^{6}, S^{7}$ are visible when they are at the end of consistency.

From the last Statement follows that visible edges of some closed polygon may be found by using the sequences Rg and Rp of the corresponding consistency.

Using the upper Statements we separate the set of the regularities to the following subsets: 
$V\left(S^{7}, S^{5}, S^{6}\right)$ the sub set of the visible regularities; $I\left(S^{1}, S^{2}, S^{3}, S^{4}, S^{8}\right)$ the subset of invisible regularities;

Let us note that visible regularities may describe also invisible and partially covered edges. Follows that the visible regularity describes invisible edge if the couple ends of regularity are invisible. If one end is visible but the other is invisible the visible regularity describes partially covered edge.

Let us denote by $X$ the length of a regularity over the axis $0 x$, and by $Y$ the length over $0 \mathrm{y}$. Then we denote by $S_{x}$ the sum of the $X$ co-ordinate of a set of regularities. Let us suppose an image containing $2 \mathrm{D}$ object, which border is approximated by closed polygon with given accuracy. We shall consider that the visibility of a part of the border is the same as the visibility of the approximating polygon edge. We give now a simple but very fast algorithm for finding of visible, invisible and partially covered edges of a closed polygon. To do this we process the sequences $\mathrm{Rg}$ and $\mathrm{Rp}$, describing the polygon's border, from left to the right. Using the Statement 2. we find all visible edges in the beginning and at the end of the polygon. Applying Statement 1. we find all invisible edges of the polygon described by invisible regularities. Finally, we have to determine all visible, invisible and partially covered edges described by visible regularities. To do this we find all sub-sequences of $\mathrm{Rg}$, which have the following type: invisible, visible IV (the sub-sequence contain first invisible regularities and after visible); visible, invisible VI (the sub-sequence contain first visible regularities and after invisible). Each sub-sequence IV we process from left to the right adding in $S_{x}$ the $x$ co-ordinate of each invisible regularity and subtract the $x$ co-ordinate of each visible. On each step we check $S_{x}$. If $S_{x}<0$ the last added regularity describe invisible edge. If $S_{x}>0$ the last added regularity describe visible or partially covered edge. If $S_{x} \geq 0$ before the last adding, then the last added regularity describe visible edge, if $S_{x}<0$ the last added regularity describe partially covered edge. We process the sub-sequences VI from right to the left using the same reasoning.

Using the last algorithm we obtain the following advantage: we find the visibility of $2 \mathrm{D}$ object border without processing the image (to do it we process the $2 \mathrm{D}$ object model). So we reduced the problem of visibility of $2 \mathrm{D}$ border to the problem of processing finite numerical sequences, with Integer elements. It allow us to increase the speed. The result of the application of the upper algorithm on Fig 2. are shown.

\section{Rotation Problem of Plane Figures}

Let us consider a closed polygon and suppose that all visible, invisible and partially covered edges are determined. Let us rotate the polygon in positive direction (anticlockwise) by angle $\varphi$.

In [8] we proved that each rotation maps a regularity in a regularity. It follow that, to find the image of a closed polygon is sufficient to find the image of the consistency describing this polygon. Therefore we have to determine the image of each regularity after applying of rotation. To do this we use the sequence An. We add the angle of rotation to each element of this sequence. From the definition of regularity follow that to each regularity corresponds just one angle. Therefore we determine the image $\mathrm{Rg}$ 
of $\mathrm{Rg}$ by using the image An. As an example let us consider the polygon shown on the Figure 2. Let us suppose that the sequence An corresponding to the regularities, which describe the polygon, is the following:

$A n=110,130,30,10,40,0,90,0,300,330,250,230,180,240$

Let us assume an angle of rotation $\varphi=50^{\circ}$ in anticlockwise direction. Then we obtain An by adding (subtracting in the case of clockwise direction) the angle to each element of the sequence An:

$\mathrm{An}^{\prime}=160,180,80,60,90,50,140,50,350,380,300,280,230,290$.

Because to each angle corresponds just one regularity we obtain the sequence:

$\mathrm{Rg}^{\prime}=5,7,3,1,3,5,3,4,3,4,6,4$. As a result $\quad \mathrm{Rp}=1,1,2,1,1,1,1,1,1,2,1,1$.

The sequence $\mathrm{Ln}$ is the same after applying the rotation.

In order to find the first point of the description of a rotated polygon we apply the formulas (2) over the first point of the regularities: $x^{\prime}=x \cdot \cos (\varphi) ; y=y \cdot \sin (\varphi)(2)$, where $(x, y)$ are the co-ordinates of the origin, and ( $\left.x^{\prime}, y^{\prime}\right)$ are the image.

In the previous paragraph we proved that only some from the regularities $S^{1}, S^{3}, S^{5}$ may be a first regularity in the consistency. Follows that, to find the first point of polygon description of the rotated polygon we have to looking for the point $\left(\mathrm{x}_{\text {min }}\right.$, $y_{\text {min }}$ ) processing the regularities $S^{1}, S^{3}, S^{5}$ from the sequence $\mathrm{Rg}^{3}$. Therefore we apply formulas (2) only over these regularities from $\mathrm{Rg}$, which have image $S^{1}, S^{3}, S^{5}$. After that we rotate $\mathrm{Rg}$ to the left so that the regularity containing the first point of description to be first in the sequence. The same number of rotations we apply over the sequences $\mathrm{Rp}, \mathrm{An}, \mathrm{Ln}$. With the next example we show a rotation in three steps to the left of a finite numerical sequence. The original sequence:

$5,7,3,3,1,3,5,3,4,3,4,4,6,4$;

becomes after three steps rotation to the left: $\quad 3,1,3,5,3,4,3,4,4,6,4,5,7,3$. By this way we find the image of the rotated polygon using the sequences $\mathrm{Rg}, \mathrm{Rp}$, An, Ln. The visible, invisible and partially covered edges we determine applying the algorithm introduced in the precede paragraph.

Our approach has the following advantage: reduce the problem for finding the image of a closed polygon after applying rotation to the problem of processing finite numerical sequences and comparing integer numbers. This technique allows us to create very fast algorithm performing rotation of closed polygons.

In [9] we describe the models of the $3 D$ objects as finite series of plane figures. It follow that the problem for finding the image of a 3D object after applying of rotation reduces to the problem of finding the images of closed polygons.

\section{Visibility of Space Triangles}

Most of the methods for reconstruction and presentation of $3 \mathrm{D}$ object use a plane triangle element for approximation of the object's surfaces. Therefore for determining the visibility of the surfaces it is sufficient to investigate the problem for visibility of a triangle in 3D space. We consider triangles whose vertices lies on two different planes cutting the 3D object surfaces. Hence a couple of vertices lie on one plane and the third on an other plane. Therefore to investigate the visibility of a triangle in $3 \mathrm{D}$ space it is sufficient to investigate the visibility of their vertices. 
Let us assume a set of planes cutting the 3D object. Let us consider that the direction of observation is along the positive direction of axis Oy. Concerning the visibility of the triangle's vertices we have the following possibilities:

1. all vertices are invisible - then we consider the corresponding triangle invisible;

2. all vertices are visible - then the triangle is visible;

3. a couple vertices are visible, one is invisible - the triangle is partially covered;

4. a couple vertices are invisible, one is visible - the triangle is partially covered.

Because in the first two cases the type of the triangle is well defined we concentrate on the last two. We denote visible vertices by $\mathrm{V}$ and invisible by $\mathrm{I}$.

Let us consider a triangle which has a pair of visible vertices and one invisible. (see Fig.3.a). From the theoretical concepts introduced in the previous paragraph it follows that one edge is visible and two are invisible. Let us denote by $A$ and $M$ the border points between the visible and invisible parts of the couple partially covered edges. We suppose that the border between $\mathrm{A}$ and $\mathrm{M}$ is approximated by straight line. Then the visible part of the triangle is the quadrangle VVMA, and the invisible part is the triangle AMI.

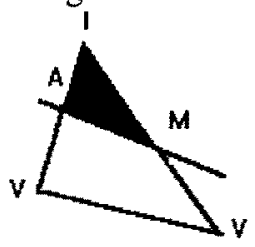

a)

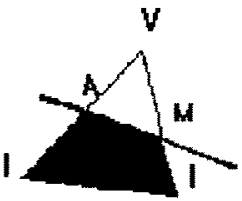

b)

Figure 3. a)Triangle with two visible and one invisible vertices.

b)Triangle with two invisible and one visible edge.

Let us consider now a triangle with two invisible edges and one visible (see Figure 3.b). This triangle has two partially covered edges and one invisible. We denote by $\mathrm{A}$ and $\mathrm{M}$ the border points between the visible and invisible parts of the couple of partially covered edges. Suppose that the border between $A$ and $M$ is approximated by straight line. It follows that the invisible part of the triangle is the quadrangle IIMA, the visible part is triangle AMV.

Using the upper concepts we reduce the problem of visibility of space triangle to the problem of visibility of the edges and finding the points $A$ and $M$.

The cases when all vertices are visible but a part of the triangle is covered may occur when the direction of observation is not parallel to the cutting planes. To find the shape of the reconstructed 3D object when the direction of observation has an angle $\alpha$ with the cutting planes we reconstruct the object when the cutting planes are parallel to the direction of observation. Then we rotate the 3D object by angle $\alpha$. To find the vertices of the covered part we rotate by angle $\alpha$ the points $A, M$ and I of the partially covered triangles. By using the image of these points we obtain the covered parts of the triangles which approximate the 3D objects surfaces..

So in the case when the surfaces of the 3D objects are approximated (represented) by triangles the problem for finding the visibility of the surfaces reduces to the problem of the visibility of triangle's vertices in the space. 


\section{Results}

On the basis of all introduced theoretical concepts a fast algorithm for determining the visible, invisible and partially covered surfaces was developed. We performed experiment to validate the correctness of this algorithm. The result is shown on Fig. 4. With the help of the software for 3D objects reconstruction represented in [10] a 3D cone is reconstructed there by using two plane sections cutting this cone. After reconstruction it is rotated on an angle equal to $50^{\circ}$ respect to the axis $\mathrm{Ox}$ and an angle $180^{\circ}$ respect to the axis $\mathrm{Oz}$. This axis is normal to the monitor screen. The nearest triangles are painted in most light and the others in dark. Let us note that we don't solve shadows problem and the problem for realistic painting of the surfaces. We use different colours to illustrate visible and partially covered triangles approximating the surfaces, and their disposition respect to the observer. Invisible triangles are not shown.
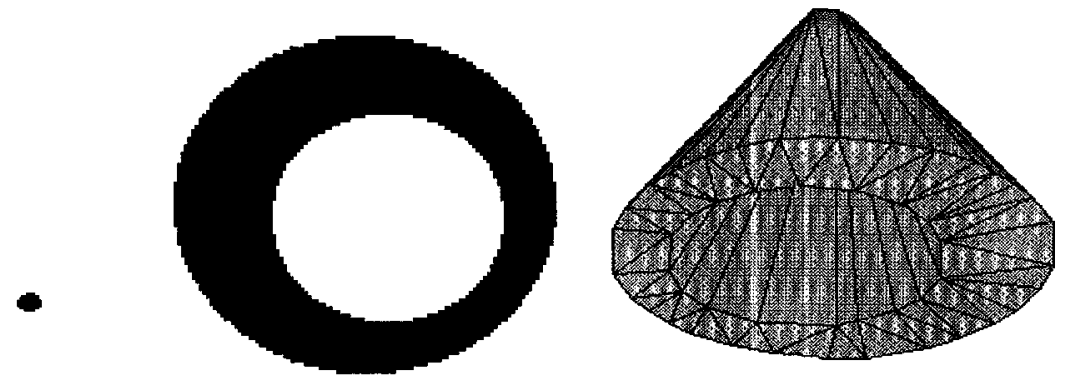

Figure 4. Two 2D sections and $3 \mathrm{D}$ reconstructed object with visible and partially covered triangles.

On the Fig. 5. we show the cone after rotation by the following angles: respect to axis $\mathrm{Ox}-40^{\circ}$; respect to $\mathrm{Oy}-\left(-30^{\circ}\right)$; respect to $\mathrm{Oz}-130$. The point of view is two times far than point of view on Fig.4.

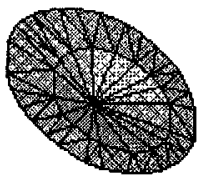

Figure 5. An other view of the cone after rotation.

In the worst case, the calculation complexity of our method and algorithm for determining the visibility of the surfaces is proportional to $O[n . k .1]$, where $n$ is the number of the plane sections cutting the 3D object's surfaces; $k$ - the number of $2 \mathrm{D}$ objects on the plane section containing maximal number of 2D objects; 1 - the number of the vertices on the 2D object with maximal number of vertices. We suppose that the 3D object may have branches.

Using our approach the following advantages may be obtained:

1.The problem for finding the visibility and the image of $3 D$ objects surfaces after applying of rotation is reduced to the problem of processing finite numerical sequences and comparing of Integer numbers; 2. High speed of performance.

Our approach is suitable for application when the $3 \mathrm{D}$ object has a complicate shape and a many triangles approximating the object's surfaces. 


\section{Conclusion}

Our research continue with the investigation of the shadow problem and the problem for painting the 3D object's surfaces. The application of a technique based on regularities and consistencies will reduce the number of calculations and will increase the speed of the algorithms solving the upper problems.

\section{References}

1.Blinn J.F., Computer Display of Curved Surfaces, Ph.D. dissertation, Dept. of Computer Science, University of Utah, 1978.

2.Badler N., O'Rourke J., Spherical Representation of a Human Body for Visualizing Movement, IEEE Proceeding, 67 (October 1979), pp.1397-1403.

3.Clark J.,Hierarchical Geometric Models for Visible Surface Algorithms, CACM, 19 (1976), pp.547-554.

4.Gitlen C., Rouke J.,Subramanian V.,On reconstructing polyhedra from parallel slices, Technical Report 25, Dept.Comput.Sciences, Smith Colledge, Northampton, MA,March, 1993.

5.Franklin W. R., A linear Exact Hidden Surface Algorithm, SIGGRAPH'80, Seattle, Wash., July 1980,pp. 117-123.

6.Hegen M.,The perception of Picture, New York: Academic Press, 1980.

7.Knowlton K., Cherry L., Atoms -A three-D opaque molecular system for colour pictures of space filling or ball and stick models, Computers \& Chemistry, 1 (1977), pp. 161-166.

8.Sirakov N, Muge F., Comparing 2D borders using regular structures, Proc. of RecPad'94, Lisbon, Portugal, 22-25.03.1994,pp.169-176.

9.N.Sirakov, Recognition of Shapes from a Finite Series of Plane Figures, NATO ASI series, Series F, Vol.126, Springer-Verlag, pp. 453-463 (1994).

10.Sirakov N., Automatic Reconstruction of 3D Branching Objects, IEEE Computer Society, Volume II Track B, Pattern Recognition and Signal Analysis, pp.620-624, 1996.

11.Rodgers J.,Algoritmic base of machine graphics, Moscow, Mir, 1989.

12.Sutherland I. E., Sproull R.F., A Characterisation of Ten Hidden-Surface Algorithms, ACM Computing Surveys, 6 (1984), pp.1-56.

13. Whitted T.,An Improved Illumination Model for Shaded Display, CACM, 23 (1980),pp. 343-349.

14.Y.F.Wang,J.K.Aggarwal, Surfaces reconstruction and representation of 3D scenes, Pattern Recognition 19, N 3,197-207,1996.

15.J.D.Boissonnat, Three-dimensional reconstruction of complex shapes based on the Delaunay triangulation, Biomedical Image Processing and Biomedical Visualisation, San-Jose CA,SPIE, Vol.1905, part 2,pp.964-975, 1993.

16.N. Ayache, Medical Computer Vision, Virtual reality and Robotics, Image and vision Computing, Vol.13, N 4. May 1995, 295-312.

17.J.Felder, N. Ayache, F.Betting, 3D-2D projective registration of free form curves and surfaces, Raport de research, N 2434, December 1994, INRIA, France. 